\title{
Evaluación de la resistencia a la corrosión de la aleación Ti6Al4V modificadas mediante oxidación térmica
}

\author{
Janer Orozco-Rodríguez ${ }^{\mathrm{a}}$, Dayron Ortiz Jaraba ${ }^{\mathrm{b}}$, Jhon Schmalbach Trujillo ${ }^{\mathrm{b}}$, Ricardo Mendoza Quiroga ${ }^{\mathrm{b}}$, \\ Carlos Soto-Montaño ${ }^{a} \&$ Roosvel Soto-Diaz ${ }^{a}$ \\ ${ }^{a}$ Facultad de Ingeniería, Ingeniería Mecánica, Universidad Autónoma del Caribe, Barranquilla, Colombia. \\ Janner.orozco@uac.edu.co, carlos.soto@uac.edu.co, roosvelt1019@gmail.com \\ ${ }^{b}$ Facultad de Ingeniería, Universidad Antonio Nariño, Puerto Colombia, Colombia. \\ rimendoza@uan.edu.co,dortiz18@uan.edu.co,jschmalbach44@uan.edu.co
}

Recibido: Abril 10, 2020.

Recibido en su versión corregida: Mayo 07, 2020.

Aceptación: Mayo 11, 2020.

Cómo citar: Orozco-Rodriguez, J., Ortiz Jaraba, D., Schmalbach Trujillo, J., Mendoza Quiroga, R., Soto-Montaño, C. \& SotoDiaz, R. (2020). Evaluación de la resistencia a la corrosión de la aleación Ti6Al4V modificadas mediante oxidación térmica. Revista Sextante, 22, pp. 33 - 39, 2020.

\section{Resumen}

En este trabajo se realizó un estudio para determinar la resistencia a la corrosión de la aleación Ti6Al4V anodizada y oxidada térmicamente para aplicaciones biomédicas. Para el estudio se realizaron ensayos de microscopía óptica (MO), microscopía electrónica de barrido, anodización (MEB), oxidación térmica, potencial a circuito abierto y curvas de polarización potenciodinámica. En el proceso de ensayo las condiciones de anodización se mantuvieron constantes en las muestras, pero variando las temperaturas de oxidación térmica en $520^{\circ} \mathrm{C}, 560^{\circ} \mathrm{C}$ y $600^{\circ} \mathrm{C}$. Los resultados mostraron inicialmente las microestructuras donde se evidenciaron que las fases $\alpha$ y $\beta$ las cuales son responsables del comportamiento mecánico, resistencia a altas temperaturas, resistencia a la corrosión y biocompatibilidad. En las pruebas de corrosión, se observaron curvas de potencial abierto con un rendimiento superior en comparación con el metal base el cual está directamente relacionado con la temperatura de oxidación térmica. En las curvas potenciodinámicas se observó que la muestra oxidada a $520^{\circ} \mathrm{C}$ presentó un comportamiento noble a la corrosión y menor tasa de corrosión que las demás muestras. Finalmente, como conclusión se determinó que las muestras oxidadas térmicamente mejoran sustancialmente la resistencia a la corrosión a diferentes temperaturas en comparación con el metal base, esto hace que aumente la biocompatibilidad para sistemas o aplicaciones biomédicas.

Palabras claves: Anodizado; Corrosión; Oxidación térmica; Ti6Al4V.

\section{Evaluation of corrosion resistance of the alloy Ti6Al4V anodized modified by thermal oxidation}

\begin{abstract}
In this paper a study was conducted to determine the corrosion resistance of the alloy Ti6Al4V anodized and thermally oxidized for biomedical applications. For the study, optical microscopy (OM), scanning electron microscopy, anodization (SEM), thermal oxidation, open circuit potential and potentiodynamic polarization curves were carried out. In the assay process conditions remained constant anodization in the samples, but varying temperatures thermal oxidation at $520^{\circ} \mathrm{C}, 560{ }^{\circ} \mathrm{C}$ and $600{ }^{\circ} \mathrm{C}$. The results initially showed the microstructures where it was evidenced that the $\alpha$ and $\beta$ phases which are responsible for the mechanical behavior, resistance to high temperatures, resistance to corrosion and biocompatibility. In corrosion tests, open potential curves were observed with superior performance compared to the base metal which is directly related to the thermal oxidation temperature. Potentiodynamic curves in it was observed that the oxidized sample at $520^{\circ} \mathrm{C}$ provided a noble corrosion resistance and lower corrosion rate than other samples. Finally, as conclusion it was determined that the samples thermally oxidized substantially improve the corrosion resistance at different temperatures compared to the base metal, this increases the biocompatibility or systems for biomedical applications.
\end{abstract}

Keywords: Anodized; Corrosion; Thermal Oxidation; Ti6Al4V. 


\section{Introducción}

El titanio es el cuarto metal estructural más abundante y sus propiedades lo han convertido en el material de elección en muchas industrias tales como la aeroespacial (alabes), biomédica (prótesis), automovilística (bielas, muelles) debido a su excelente resistencia mecánica y su resistencia a la corrosión en una amplia variedad de entornos. La formación de una fina película de óxido pasivo auto adherente sobre sus superficies se considera responsable de este atributo. Sin embargo, la velocidad de corrosión del titanio y sus aleaciones es significativa cuando se expone a medios agresivos (Hernandez Lopez, 2015; Guleryuz \& Cimenoglu, 2004; Gil \& Planell, 1993). El rápido aumento del uso del titanio y sus aleaciones ha llevado a la investigación sobre su comportamiento a la corrosión, dado que la película pasiva es la responsable de la excelente resistencia, por lo tanto, cualquier tratamiento o modificación que facilite su formación y engrosamiento ofrecerá un mejor comportamiento a la corrosión (Guleryuz \& Cimenoglu, 2004).

En estos últimos años la fabricación de piezas mecánicas, electrónicas y biomédicas con aleaciones de titanio han venido en aumento y paralelamente el crecimiento de las investigaciones de cómo mejorar las propiedades de dicho metal. Las aplicaciones del titanio son muchas y por ese motivo se ha buscado la forma de aplicar tratamientos fisicoquímicos con el fin de optimizar su superficie y modificar su capa de óxido para un mejor comportamiento a la corrosión. La formación de nanoestructuras en la capa de óxido de titanio ha sido una alternativa notable en el comportamiento de diversos dispositivos tales como los implantes médicos, celdas solares, dispositivos electrocrómicos (Hernandez Lopez, 2015).

La modificación superficial es un método prometedor para aumentar la dureza superficial, la resistencia a la corrosión y la resistencia al desgaste del titanio y sus aleaciones (Concha-Guzman, Gonzalez, \& Cuevas-Arteaga, 2012). Existen una gran variedad de métodos entre los cuales se encuentran el proceso sol-gel, las técnicas hidrotérmicas y la anodización para mejorar el crecimiento de la capa de óxido del titanio y dependiendo de su tamaño y área superficial será su eficiencia, por ello la importancia del estudio del procedimiento experimental en el crecimiento autoorganizado de nanoestructuras. De los procesos mencionados anteriormente la anodización electroquímica es el método más usado para obtener estructuras nanoporosas o nanotubulares (ConchaGuzman, Reynaud Morales, \& Cuevas-Arteaga, 2010).

Para muchas de estas aplicaciones, es crucial maximizar el área superficial específica para lograr una eficiencia global máxima. En la formación de nanotubos se genera una película de óxido que mejora la resistencia a la corrosión del titanio, pero si se quiere aumentar el espesor de dicha capa existe un tratamiento superficial complementario llamado oxidación térmica (Roy, Berger, \& Schmuki, 2011).

Revisando la literatura se encuentran distintos valores de la resistencia a la corrosión en las aleaciones de titanio, pero normalmente emplean extensas horas de sostenimiento en el tratamiento y con diferentes temperaturas, debido a esto se requiere estudiar este tratamiento con variaciones en la temperatura y tiempo (Cely, Castellar, Pereira, \& Ángel, 2018). El ámbito de sistemas que aumenten la resistencia a la corrosión de los materiales utilizados en diferentes ámbitos industriales ha aumentado de manera vertiginosa. En este caso las aleaciones de Titanio tienen una aplicación biomédica importante la cual ha hecho imperante el uso de técnicas de protección de diferentes formas con el objeto de mejorar esa resistencia a la corrosión y beneficio biológico.

El objetivo de este trabajo es evaluar la resistencia a la corrosión de la aleación Ti6Al4V anodizada y oxidada térmicamente. El trabajo propuesto logra muestras libres de defectos anodizadas y oxidadas térmicamente a diferentes temperaturas, desarrollando análisis microestructurales y ensayos de corrosión potenciodinámicos.

El siguiente artículo está organizado de la siguiente manera: Se exponen en la sección 2 los materiales y métodos desarrollado para esta investigación. Posteriormente, Resultados y discusión se presentan en la sección 3. Finalmente, en la sección 4 se explican las principales conclusiones, así como las futuras experimentaciones de este trabajo. 


\section{Materiales y métodos}

\subsection{Material}

El metal utilizado en el procedimiento de anodización y oxidación térmica fue una aleación de titanio Ti6Al4V con un espesor de $3 \mathrm{~mm}$ y una composición química que se muestra en la Tabla 1 para analizar el comportamiento inicial.

\subsection{Anodizado}

Antes del proceso de anodizado, las muestras se pulieron mecánicamente con alúmina $(0,05 \mu \mathrm{m})$ y se atacaron químicamente con ácido Nital diluido al $3 \%$ para eliminar la capa superficial de óxido en las muestras que se forma espontáneamente antes de aplicar el proceso electroquímico. Posteriormente, se realizó una limpieza ultrasónica en un equipo marca Elma (E $30 \mathrm{H}$ Elmasonic technologies $®)$ por espacio de 10 minutos con etanol. Para el proceso de anodizado se realizó el decapado con el mismo electrolito de trabajo. La muestra la composición química utilizada para el decapado de las muestras: $\mathrm{H} 3 \mathrm{PO} 4+0,2 \%$ en peso de $\mathrm{HF} 1 \mathrm{M}$ y ejecutada durante un tiempo de 60 segundos. Las muestras se enjuagaron en agua destilada y se secaron al aire. Luego, se llevó a cabo la anodización en una celda de dos electrodos a un voltaje constante de $14 \mathrm{~V}$ en un electrolito de $\mathrm{H} 3 \mathrm{PO} 4+0,2 \%$ en peso de HF $1 \mathrm{M}$ a $25^{\circ} \mathrm{C}$ durante 60 minutos. Se utilizó una placa de acero inoxidable AISI-SAE304 como cátodo. (Ver Tabla 2)

\subsection{Oxidación térmica}

Para el proceso de oxidación térmica, las muestras se trataron térmicamente a temperaturas de $520^{\circ} \mathrm{C}, 560^{\circ} \mathrm{C}$ y $600^{\circ} \mathrm{C}$ con una velocidad de calentamiento de $5^{\circ} \mathrm{C} / \min$ y un tiempo de mantenimiento posterior de 2 horas con una velocidad de enfriamiento en el horno de $2^{\circ} \mathrm{C}$ por minuto. Las muestras se sacaron del horno aproximadamente 24 horas después de iniciado el proceso. En la Tabla 3 se consolidan los experimentos realizados.

\subsection{Ensayos de corrosión}

Las mediciones de potencial de circuito abierto y polarización potenciodinámica de la aleación Ti6Al4V y muestras modificadas en la superficie se
Tabla 1. Composición química del Ti6Al4V.

\begin{tabular}{c|cccccccc}
\hline Ti6 A 14 V & Al & $\mathbf{V}$ & $\mathbf{F e}$ & $\mathbf{C}$ & $\mathbf{O}$ & $\mathbf{N}$ & $\mathbf{H}$ & $\mathbf{T i}$ \\
\hline $\begin{array}{c}\text { UNE-7301 } \\
\text { IS O 5832 } \\
\mathbf{3}\end{array}$ & $5.5-6.50$ & $3.5-4.50$ & 0.25 & 0.08 & 0.13 & 0.05 & 0.01 & $\mathrm{Bal}$ \\
$\begin{array}{c}\text { A S TM } \\
\text { F 136 }\end{array}$ & $5.5-6.75$ & $3.5-4.50$ & 0.30 & 0.08 & 0.20 & 0.05 & 0.01 & $\mathrm{Bal}$ \\
\hline
\end{tabular}

Fuente: Los autores.

Tabla 2. Condiciones de anodizado.

\begin{tabular}{cc}
\hline Tipo & Potenciostático \\
\hline Electrolito & $\begin{array}{c}1 \mathrm{M} \mathrm{H}_{3} \mathrm{PO}_{4}+ \\
0.2 \mathrm{wt} \% \mathrm{HF}\end{array}$ \\
\hline Temperatura & Ambiente \\
\hline Ánodo & Ti6Al4V \\
\hline Cátodo & Acero inoxidable \\
& 304 \\
\hline Voltaje & $14 \mathrm{~V}$ \\
\hline Tiempo & $1 \mathrm{hora}$ \\
\hline $\begin{array}{c}\text { Distancia entre } \\
\text { electrodos }\end{array}$ & $20 \mathrm{~mm}$ \\
\hline
\end{tabular}

Fuente: Los autores.

Tabla 3. Parámetros del proceso de oxidación térmica.

\begin{tabular}{ccccc}
\hline Probeta & Temperatura & $\begin{array}{c}\text { Tiempo de } \\
\text { Sostenimiento }\end{array}$ & $\begin{array}{c}\text { Velocidad de } \\
\text { Calentamiento }\end{array}$ & $\begin{array}{c}\text { Velocidad de } \\
\text { enfriamiento }\end{array}$ \\
\hline 1 & $520^{\circ} \mathrm{C}$ & $2 \mathrm{H}$ & $5^{\circ} \mathrm{C} / \mathrm{min}$ & $2^{\circ} \mathrm{C} / \mathrm{min}$ \\
2 & $520^{\circ} \mathrm{C}$ & $2 \mathrm{H}$ & $5^{\circ} \mathrm{C} / \mathrm{min}$ & $2^{\circ} \mathrm{C} / \mathrm{min}$ \\
3 & $560^{\circ} \mathrm{C}$ & $2 \mathrm{H}$ & $5^{\circ} \mathrm{C} / \mathrm{min}$ & $2^{\circ} \mathrm{C} / \mathrm{min}$ \\
4 & $560^{\circ} \mathrm{C}$ & $2 \mathrm{H}$ & $5^{\circ} \mathrm{C} / \mathrm{min}$ & $2^{\circ} \mathrm{C} / \mathrm{min}$ \\
5 & $600^{\circ} \mathrm{C}$ & $2 \mathrm{H}$ & $5^{\circ} \mathrm{C} / \mathrm{min}$ & $2^{\circ} \mathrm{C} / \mathrm{min}$ \\
6 & $600^{\circ} \mathrm{C}$ & $2 \mathrm{H}$ & $5^{\circ} \mathrm{C} / \mathrm{min}$ & $2^{\circ} \mathrm{C} / \mathrm{min}$ \\
\hline
\end{tabular}

Fuente: Los autores.

llevaron a cabo utilizando una estación de trabajo electroquímica Gamry 600®. El montaje de las probetas en la celda electroquímica se realizó con electrodo de referencia de $\mathrm{Ag} / \mathrm{AgCl}$ a $3 \mathrm{M} \mathrm{KCl}$ y contra electrodo de platino. Las curvas comenzaron desde $-0.3 \mathrm{~V}$ (vs OCP) a $+3 \mathrm{~V}$ (vs. $\mathrm{Ag} / \mathrm{AgCl}, \mathrm{KCl}$ saturado), y el escaneo inverso de $+3 \mathrm{~V}$ (vs. $\mathrm{Ag} / \mathrm{AgCl}, \mathrm{KCl}$ saturado) a $+1.3 \mathrm{~V}$ (vs $\mathrm{Ag} / \mathrm{AgCl}, \mathrm{KCl}$ saturado) a una velocidad de exploración constante de $0,16 \mathrm{mV} / \mathrm{s}$. Se usó la densidad de corriente de corrosión $\left(\mathrm{A} / \mathrm{cm}^{2}\right)$ para determinar la cinética de corrosión de las diferentes muestras. 


\subsection{Microscopía electrónica de barrido}

Para la evaluación de los ataques generados por los ensayos de corrosión, la formación de capas de oxido y las morfologías de estas se utilizó un microscopio electrónico de barrido marca JEOL 5910LV con una intensidad de $15 \mathrm{kV}$ para el metal base y las probetas oxidadas térmicamente.

\section{Resultados y discusión}

\subsection{Caracterización microestructural}

En la Figura 1 se observan las micrografías de la aleación Ti6Al4V antes del proceso de modificación superficial. En la sección (a) se muestra la sección longitudinal de la micrografía tomada a la probeta en su caracterización microestructural y la sección (b) se observa la sección transversal de la aleación de titanio. En estas se evidenciaron diferencias significativas conforme a las características que se presentaron y que dependieron según el tipo de corte realizado, se distinguió una matriz $\alpha$ (alfa) de color blanco y la fase $\beta$ (beta) contenida de color gris oscuro.

La sección longitudinal (a) de la Figura 1 se aprecian granos de forma alargada, debido a la deformación a la que se ve sometida el material en su proceso de fabricación, mientras que en la la micrografía de la sección transversal (b) se presenta una forma de grano asemejado a la morfología equiaxial y con una estructura uniforme durante todo su proceso en forma de dendritas con límites de granos lo bastante gruesos en sus terminaciones. Esto permite generar una relación entre los resultados con la microestructura de esta misma aleación que se encuentra en investigaciones (Yang, Yang, Yu, Zeming, \& Zeng, 2017).

La importancia de tal material seleccionado dentro de la investigación, en comparación con otros que se encuentren en su misma categoría, se muestra relevante tal aspecto debido al análisis de sus principales características a nivel microestructural, destacando que la estructuras cristalinas $\alpha$ y $\beta$ tienen una excelente resistencia mecánica y propiedades favorables a temperaturas elevadas, donde no solo pueda evaluarse este factor, sino también a otros posibles factores que involucren su resistencia a la corrosión y biocompatibilidad para posteriores investigaciones.

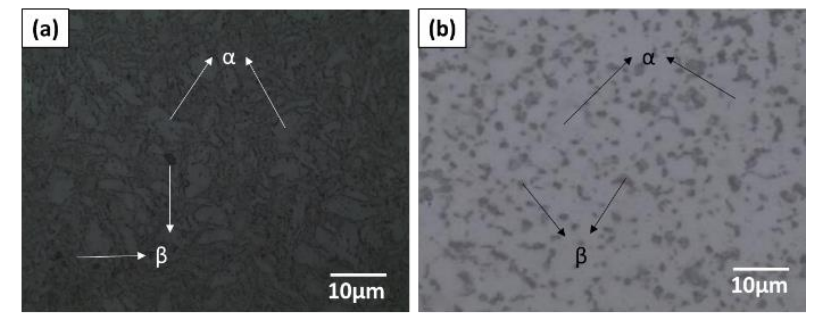

Figura 1. Microestructura de la aleación antes del proceso de modificación superficial

Fuente: Los autores.

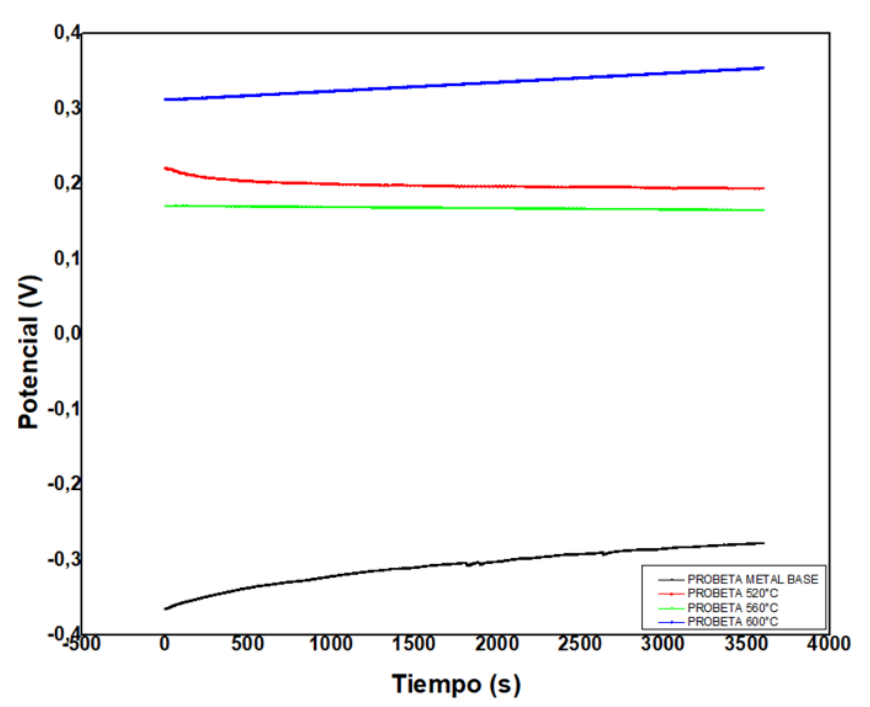

Figura 2. Potencial a circuito abierto de las muestras modificadas superficialmente y del metal base.

Fuente: Los autores.

\subsection{Potencial a circuito abierto}

La Figura 2 muestra los potenciales a circuito abierto de las muestras oxidadas térmicamente y el metal base sin tratar. Inicialmente la condición que mayor aumento su potencial fue la PROBETA $600^{\circ} \mathrm{C}$, por otro lado, se observan valores estables de los potenciales a circuito abierto de los materiales modificados $\left(520^{\circ} \mathrm{C}, 560^{\circ} \mathrm{C}\right.$ y $\left.600^{\circ} \mathrm{C}\right)$ que van entre $0,167 \mathrm{~V}$ y $0,332 \mathrm{~V}$ para las diferentes condiciones. Los valores inician con el METAL BASE que presentó un valor de $-0,308 \mathrm{~V}$ promedio similar al estudiado por (Longhitano, y otros, 2018) que fue promedio de $-0,30 \mathrm{~V}$.

Los valores de potencial de circuito abierto en los materiales modificados superficialmente aumentaron ampliamente respecto al metal base como se muestra en la Figura 2 lo que representa una gran mejora en el comportamiento a la corrosión respecto al metal base. Lo anterior se debe a que durante la anodización y oxidación térmica se generó un aumento en la formación de la capa 
porosa de $\mathrm{TiO} 2$ en la superficie de la muestra de titanio, el cual cambia su potencial con respecto al metal base, moviéndose hacia una dirección noble, lo que incrementa su resistencia a la corrosión (Yang, Yang, Yu, Zeming, \& Zeng, 2017).

\subsection{Curvas potenciométricas}

En la Figura 3 y la Tabla 4 se muestran las curvas potenciodinámicas y los datos obtenidos del análisis tafel respectivamente. En la Figura 3 podemos observar que los potenciales de las probetas son variados respecto a las temperaturas de modificación, sin embargo, están por encima de la línea 0 lo que da a entender una mejora considerable respecto a la corrosión del metal base como lo muestra (Longhitano, y otros, 2018; Yang, Yang, Yu, Zeming, \& Zeng, 2017). Los mejores potenciales de corrosión (Ecorr) son los presentados por las PROBETA $520^{\circ} \mathrm{C}$ y PROBETA $600^{\circ} \mathrm{C}$, pero la $520^{\circ} \mathrm{C}$ presenta un mejor potencial potenciodinámico, es decir, las reacciones en función anódica y catódica mejoran al ubicarse más arriba (mayores potenciales) y a la izquierda (menores densidades de corriente). Adicionalmente las curvas muestran que en la región anódica de las curvas de polarización se presenta una transición activa-pasiva en todos los casos de oxidación térmica (Cely, Castellar, Pereira, \& Ángel, 2018). A lo anterior se evidencia que la región activa de las curvas de polarización de las muestras modificadas superficialmente se extiende hacia la región de corriente más baja, lo que explica que la capacidad de la capa de óxido formada térmicamente ofrece una mejora en la resistencia a la corrosión (Ribeiro, Santos, \& de Sousa, 2019).

Lo anterior reafirma que la temperatura idónea para la implementación del crecimiento de la capa de $\mathrm{TiO} 2$ ideal es la de $520^{\circ} \mathrm{C}$. Trabajos como el realizado por (Cely, Castellar, Pereira, \& Ángel, 2018) muestran que las temperaturas bajas para la modificación de las capas de TiO2 en Ti6Al4V son más idóneas.

\subsection{Microscopía electrónica de barrido}

En la Figura 4 se muestra el análisis de microscopía electrónica de barrido de las superficies de las muestras modificadas mediante oxidación térmica. Se puede observar que existe un crecimiento de una capa de oxido en las superficies

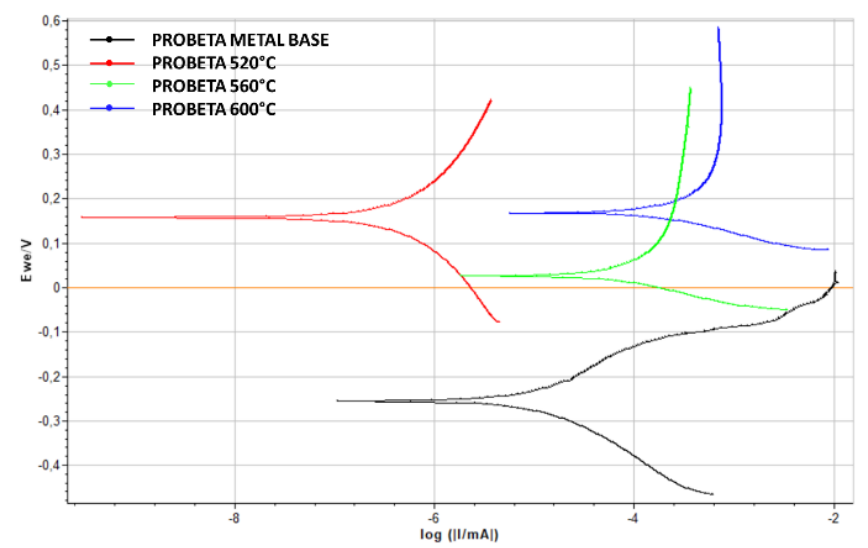

Figura 3. Curvas potenciodinámicas de las muestras modificadas superficialmente y del metal base.

Fuente: Los autores.

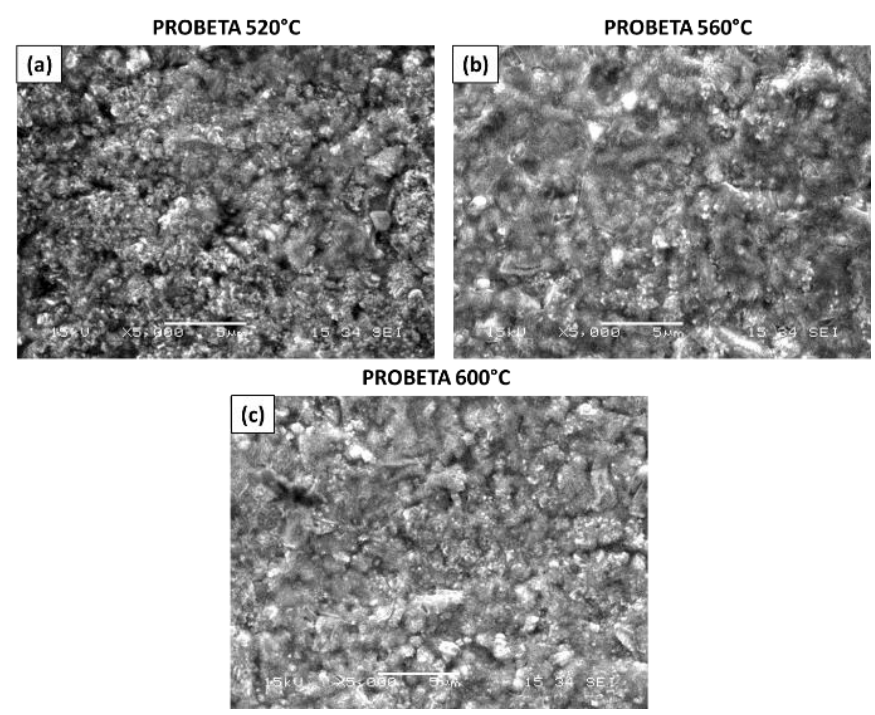

Figura 4. Microscopía electrónica de barrido de las probetas oxidadas térmicamente.

Fuente: Los autores.

Tabla 4. Datos finales de las pruebas.

\begin{tabular}{cccc}
\hline $\begin{array}{c}\text { Condición de } \\
\text { oxidación } \\
\text { térmica }\end{array}$ & $\begin{array}{c}\text { Potencial de } \\
\text { corrosión } \\
\text { Ecorr }(\boldsymbol{V})\end{array}$ & $\begin{array}{c}\text { Intensidad de } \\
\text { corriente } \\
\text { Icor }\left(\boldsymbol{u A} / \text { cm }^{2}\right)\end{array}$ & $\begin{array}{c}\text { Velocidad de } \\
\text { corrosión } \\
\text { (mpy) }\end{array}$ \\
\hline N/A & $-0,2546$ & 0,004 & $2,71558 \times 10-3$ \\
$520^{\circ} \mathrm{C}$ & 0,1579 & 0,000557 & $0,37815 \times 10-3$ \\
$560^{\circ} \mathrm{C}$ & 0,0265 & 0,04 & 0,02715 \\
$600^{\circ} \mathrm{C}$ & 0,168 & 0,104 & 0,07006 \\
\hline
\end{tabular}

Fuente: Los autores.

de las muestras tratadas térmicamente. Se observa que la capa generada no es la correspondiente a nanotubos de $\mathrm{TiO} 2$, sin embargo, se observa que el crecimiento es el de una capa porosa rica en $\mathrm{TiO} 2$. Esta capa porosa rica en $\mathrm{TiO} 2$ tiene una tendencia a aumentar el comportamiento de la corrosión 
sustancialmente respecto al metal desnudo como se evidenció en las curvas de polarización potenciodinámica.

Por otro lado, en la Figura 3 podemos observar que en las PROBETA $520^{\circ} \mathrm{C}$ se presentó un mayor crecimiento de la capa porosa respecto a las otras condiciones debido que el crecimiento de esta es mayor que en las otras condiciones. Por su parte Cely et al. (Cely, Castellar, Pereira, \& Ángel, 2018) mostró que los crecimientos de las capas porosas o películas de oxido aparte de generar aumentos en el comportamiento a la corrosión también genera un aumento en las propiedades mecánicas del material como la dureza.

\section{Conclusiones}

La microestructura de la aleación Ti6Al4V presentó las fases $\alpha$ y $\beta$ con una orientación definida, con granos equiaxiales y eso se transmite en el comportamiento de la aleación respecto a la resistencia a la corrosión. Lo anterior no solo representa un aumento en la resistencia a la corrosión, sino que estas fases aumentan las propiedades mecánicas de las muestras, trabajos a altas temperaturas y excelente biocompatibilidad debido a que propagan el crecimiento de capas de $\mathrm{TiO} 2$.

Los ensayos de corrosión de mostraron que la resistencia la corrosión de las muestras modificadas superficialmente respecto al metal base tuvo una mejora considerablemente buena. Lo anterior se debe directamente a la oxidación térmica debido a que se propagó el crecimiento de las capas de óxido de $\mathrm{TiO} 2$ sobre toda la superficie de la muestra y así mismo elevó su resistencia a la corrosión. La muestra que se trabajó a temperatura de $520^{\circ} \mathrm{C}$ presentó una mejor resistencia a la corrosión y una baja tasa de corrosión.

La microscopía electrónica de barrido mostró la generación de una capa porosa característica de un tratamiento térmico sobre la aleación de titanio (Ti6Al4V). Esta capa de oxido porosa rica en $\mathrm{TiO} 2$ corroboró los comportamientos observados en las pruebas de corrosión debido a que se pudo correlacionar dichos comportamientos con las morfologías obtenidas mediante la microscopía.
Para futuros estudios se sugiere disminuir las distancias entre las temperaturas de los tratamientos de oxidación térmica. Así como también se sugieren técnicas que permitan determinar los productos de corrosión y así generar una mejor correlación entre las curvas, gráficas y productos generados.

\section{Referencias}

Cely, M.-M., Castellar, G., Pereira, J., \& Ángel, R. (2018). Efecto de la velocidad de calentamiento sobre las propiedades mecánicas y resistencia a la corrosión de aleaciones de titanio modificadas. Ingeniare. Revista chilena de ingeniería, 577-584.

Concha-Guzman, M.-O., Gonzalez, R., \& CuevasArteaga, C. (2012). omparación de técnicas electroquímicas dinámicas y estáticas en la elaboración de nanoestructuras de TiO2 a bajo potencial. Superficie y Vacío, 25, 1-13.

Concha-Guzman, M.-O., Reynaud Morales, A., \& Cuevas-Arteaga, C. (2010). Crecimiento De Nanoestructuras De TIO2 Obtenidas Por Reacción Anódica En Soluciones Ácidas H2SO4/HF. Superficies y Vacío, 183-187.

Gil, F., \& Planell, J. (1993). Aplicaciones biomédicas del titanio $v$ sus aleaciones. Biomecánica-Originales, 34-42.

Guleryuz, H., \& Cimenoglu, H. (2004). Effect of thermal oxidation on corrosion and corrosionwear behaviour of a Ti-6Al-4V alloy. Biomaterials, 3325-3333.

Hernandez Lopez, J. M. (2015). Funcionalización superficial de aleaciones de titanio mediante anodizado para aplicaciones biomédicas. Madrid: Universidad Autónoma de Madrid.

Longhitano, G., Maria-Angeles, A., Conde, A., Larosa, M., André, J., Jardiní, A., . . . Damborenea, J. (2018). Heat treatments effects on functionalization and corrosion behavior of Ti-6Al-4V ELI alloy made by additive manufacturing. Alloys and Compounds, 961968.

Ribeiro, C., Santos, A., \& de Sousa, A. (2019). Cyclic Thermal Oxidation Evaluation to Improve 
Ti6Al4V Surface in Applications as Biomaterial. Journal of Materials Engineering and Performance, 1-7.

Roy, P., Berger, S., \& Schmuki, P. (2011). TiO2 Nanotubes: Synthesis and Applications. NanoScience, 2904-2939.

Yang, J., Yang, H., Yu, H., Zeming, W., \& Zeng, X. (2017). Corrosion Behavior of Additive Manufactured Ti-6Al-4V Alloy in NaCl Solution. Metallurgical and materials transactions A, 1 11. 